\title{
Cosmological chirality and magnetic fields from parity violating particle decays
}

\author{
Tanmay Vachaspati® ${ }^{1}$ and Alexander Vilenkin ${ }^{2}$ \\ ${ }^{1}$ Physics Department, Arizona State University, Tempe, Arizona 85287, USA \\ ${ }^{2}$ Institute of Cosmology, Department of Physics and Astronomy, Tufts University, \\ Medford, Massachusetts 02155, USA
}

(Received 1 February 2021; accepted 30 April 2021; published 20 May 2021)

\begin{abstract}
We estimate the chirality of the cosmological plasma due to parity violating decays of Standard Model particles, focusing on the example of tau leptons. The nontrivial chirality is however too small to make a significant contribution to the cosmological magnetic field via the chiral-magnetic effect.
\end{abstract}

DOI: 10.1103/PhysRevD.103.103528

\section{INTRODUCTION}

The last few decades have seen growing interest in cosmic magnetic fields on several fronts [1]. Several ideas have been proposed that can generate magnetic fields in cosmology, some of which are directly tied to known particle physics [2-4] and its possible extensions [1,5-8]. The magneto-hydrodynamical (MHD) evolution of cosmological magnetic fields is now understood quite well on the basis of analytical arguments $[9,10]$ and direct simulations [11]. There are claims for an indirect lower bound on the cosmological magnetic field strength [12-16], though not without debate $[17,18]$, and more direct evidence [19]. Concurrently there are claims of a parity violating signature that can be used to measure the magnetic field helicity spectrum $[20,21]$ though with no significant detections as yet $[22,23]$.

In parallel to these developments, motivated by heavyion collision experiments [24], there has been renewed interest in chiral effects in plasmas, namely the chiralmagnetic [25] and chiral-vortical [26] effects (CME and CVE respectively). The CME and CVE have also been applied to the evolution of cosmological and astrophysical magnetic fields [5,27-33]. In this paper we discuss how CME and CVE can effectively arise in standard cosmology with standard particle interactions due to the parity-violating decays of heavy leptons and quarks. The basic idea is that the Standard Model has a number of unstable particles that decay at various cosmological epochs, primarily due to the weak interactions. Since the weak interactions violate parity, the decay products are chiral and this provides a net

Published by the American Physical Society under the terms of the Creative Commons Attribution 4.0 International license. Further distribution of this work must maintain attribution to the author(s) and the published article's title, journal citation, and DOI. Funded by SCOAP . particle helicity to the cosmological plasma. The net particle helicity in principle leads to electric currents via the CME that can generate magnetic helicity. However, accounting only for decays of Standard Model particles, the net particle helicity is too small to significantly affect cosmological magnetic fields and their helicity.

We start by describing the physical effect in some detail in the context of the tau lepton in Sec. II, where we also estimate the induced electric currents. We find an upper bound to the magnetic helicity that can be generated due to chiral effects in Sec. III. Our conclusions are summarized and discussed in Sec. IV.

\section{CHIRALITY PRODUCTION IN TAU DECAYS}

To illustrate the physics of the effect, in this section we will discuss the decay of tau leptons in the background of a magnetic field and fluid vorticity. Except for small differences, the physics carries over to the case of decays of other particles.

\section{A. Particle decay}

Tau leptons decay into electrons (or muons) and neutrinos

$$
\tau^{-} \rightarrow e^{-}+\nu_{\tau}+\bar{\nu}_{e}
$$

and antitau into positrons and neutrinos

$$
\tau^{+} \rightarrow e^{+}+\bar{\nu}_{\tau}+\nu_{e} .
$$

These decays violate parity since they proceed primarily by the weak interactions. Therefore the tau predominantly decays into a relativistic left-handed electron, while an antitau decays into a relativistic right-handed positron. Due to the lepton asymmetry of the Universe there are more taus 
than antitaus, and the cosmological plasma gains net lefthanded chirality as taus decay.

The decay product electrons are chiral since they are produced by the weak interactions, but chirality is not preserved for massive particles. Instead, as emphasized in Ref. [34] in the context of supernovae and neutron stars, chirality is nearly equal to helicity for ultrarelativistic particles, so it is better to think of the final electrons as being in a definite helicity state. Helicity can only change due to particle interactions. We shall adopt this view in what follows.

The $\tau$ mass is $m_{\tau}=1777 \mathrm{MeV}$ and the $\tau$ lifetime in its rest frame is $\tau_{\tau}=2.9 \times 10^{-13} \mathrm{~s}$. However, the decaying taus are constantly reproduced by reactions inverse to (1), (2), ${ }^{1}$ so the number density of taus, $n_{\tau}$, remains comparable to that of photons until the time

$$
t_{\tau} \sim 10^{-7} \mathrm{~s},
$$

when the cosmic temperature drops to $T \sim m_{\tau}$. At later times $n_{\tau}$ decreases exponentially.

The particle helicity density, $n_{\chi}$, is produced in tau decays and is dissipated by helicity flipping scatterings and due to the chiral anomaly. The latter is proportional to $\alpha^{3} B^{2}$ [35], where $\alpha \approx 1 / 137$ is the fine structure constant and $B$ is the magnetic field strength, and is much slower than helicity flipping scatterings for vanishing or weak magnetic fields. We will ignore the anomalous flipping for now but will discuss it in Sec. III when we consider the effect of particle chirality on the generation of magnetic fields. The evolution of particle helicity density can be described by the kinetic equation in the relaxation time approximation,

$$
\frac{d}{d t}\left(a^{3} n_{\chi}\right)=\frac{a^{3}}{\tau_{d}}\left(\delta n_{\tau}-\delta n_{\tau}^{\mathrm{eq}}\right)-\frac{a^{3} n_{\chi}}{\tau_{\chi}},
$$

where

$$
\delta n_{\tau}=n_{\tau}^{+}-n_{\tau}^{-},
$$

$n_{\tau}^{-}$and $n_{\tau}^{+}$are the densities of tau and antitau particles, respectively, $\delta n_{\tau}^{\mathrm{eq}}$ is the equilibrium value of $\delta n_{\tau}, \tau_{d} \sim$ $\left(T / m_{\tau}\right) \tau_{\tau}$ is the decay time of taus (assuming that $T>m_{\tau}$ and with time dilation taken into account) and $\tau_{\chi}^{-1}$ is the electron helicity flipping rate. At $T \gg m_{e}$, the helicity flipping rate is suppressed by a factor $m_{e}^{2} / T^{2}$ compared to the scattering rate $\alpha^{2} T$ and a correction factor of $\alpha^{-1}$ due to infrared effects [36] (earlier estimates of the flipping rate were suppressed by another factor of $\alpha$ [34]),

\footnotetext{
${ }^{1}$ Tau particles are also produced and destroyed in scattering reactions like $\tau^{-}+\nu_{e} \rightarrow e^{-}+\nu_{\tau}$. We disregard them in what follows, since they do not change the order of magnitude of the effect.
}

$$
\tau_{\chi} \sim \frac{1}{\alpha T} \frac{T^{2}}{m_{e}^{2}}
$$

The excess of antitaus over taus, $\delta n_{\tau}$, decreases due to tau decay and is described by the equation

$$
\frac{d}{d t}\left(a^{3} \delta n_{\tau}\right)=\frac{a^{3}}{\tau_{d}}\left(\delta n_{\tau}^{\mathrm{eq}}-\delta n_{\tau}\right)
$$

At temperatures below the electroweak phase transition, $T \lesssim T_{\mathrm{EW}} \sim 100 \mathrm{GeV}$, we have $\tau_{d} \ll t$, where $t$ is the cosmic time. ${ }^{2}$ This means that the equilibrium density of taus establishes very quickly (compared to the Hubble time), and the approximate solution of (7) is $\delta n_{\tau} \approx \delta n_{\tau}^{\mathrm{eq}}$. Inserting (7) in (4) and then using $\delta n_{\tau} \approx \delta n_{\tau}^{\text {eq }}$ we have

$$
\frac{d}{d t}\left(a^{3} n_{\chi}\right)=-\frac{d}{d t}\left(a^{3} \delta n_{\tau}^{\mathrm{eq}}\right)-\frac{a^{3} n_{\chi}}{\tau_{\chi}}
$$

With a given $\delta n_{\tau}^{\mathrm{eq}}$, this equation can be solved in quadratures, but we shall instead find an approximate solution. Since we are in the regime where $\tau_{\chi} \ll t$, the term on the left-hand side can be neglected and we obtain

$$
n_{\chi} \approx-\tau_{\chi} T^{3} \frac{d}{d t}\left(\frac{\delta n_{\tau}^{\mathrm{eq}}}{T^{3}}\right)
$$

where we have used $a T \approx$ const.

Once we determine the equilibrium excess of antitaus over taus, denoted by $\delta n_{\tau}^{\mathrm{eq}}$, we can estimate the chirality density of the Universe due to tau decays using (9).

\section{B. Equilibrium density}

The equilibrium density $\delta n_{\tau}^{\text {eq }}$ is given by

$\delta n_{\tau}^{\mathrm{eq}}=\frac{1}{2 \pi^{2}} \int_{0}^{\infty} d p p^{2}\left[f\left(\frac{E-\mu_{\tau}}{T}\right)-f\left(\frac{E+\mu_{\tau}}{T}\right)\right]$,

where $f(x)=\left(e^{x}+1\right)^{-1}$ is the Fermi distribution, $E=\sqrt{p^{2}+m_{\tau}^{2}}$, and $\mu_{\tau}$ is the chemical potential of $\tau$ particles. At $T \gg m_{\tau}, \mu_{\tau}$ we can expand the integrand in Eq. (10) in powers of $m_{\tau}^{2} / p^{2}$ and $\mu_{\tau} / T$. The integrations are then easily performed and we find

$$
\delta n_{\tau}^{\mathrm{eq}} \approx \frac{\mu_{\tau} T^{2}}{6}\left(1-\frac{3 m_{\tau}^{2}}{2 \pi^{2} T^{2}}\right) .
$$

We assume that the baryon and/or lepton asymmetry of the Universe was generated at $T \gg T_{\mathrm{EW}}$ by some interactions beyond the Standard Model, for example by

\footnotetext{
${ }^{2}$ This is easily verified using the relation $t \sim m_{\mathrm{P}} / \sqrt{N} T^{2}$, where $m_{\mathrm{P}}$ is the Planck mass and $N$ is the number of particle species in equilibrium.
} 
$(B-L)$-violating leptoquark decays. This asymmetry was then redistributed between the Standard Model leptons and quarks by sphaleron processes. Let us denote the lepton-tophoton ratio at $T \ll T_{\mathrm{EW}}$ by $\eta_{L}$. Generically we expect the chemical potentials of light baryons and leptons to be of the same order, and $\mu_{\tau} / T \sim \eta_{L} \sim \eta_{B}$ [37,38], where $\eta_{B} \sim 10^{-9}$ is the observed baryon-to-photon ratio. However, observations allow for $\eta_{L}$ to be much larger, with an upper bound derived from big bang nucleosynthesis of $\eta_{L} \lesssim 10^{-2}$ [39]. In the high-temperature regime, when $T$ is large compared to all relevant particle masses, we have $\mu_{\tau} / T \approx$ const, with a mass correction $\mathcal{O}\left(\mathrm{m}^{2} / T^{2}\right)$ [40]. Then Eq. (11) becomes

$$
\frac{\delta n_{\tau}^{\mathrm{eq}}}{T^{3}} \approx C \eta_{L}-K \eta_{L} \frac{m_{\tau}^{2}}{T^{2}}
$$

where $C$ and $K$ are $\mathcal{O}(1)$ numerical constants. ${ }^{3}$ The mass correction term in (12) can be qualitatively understood as follows. As the temperature decreases, it becomes energetically favorable to transfer the conserved $\tau$-lepton number from $\tau$ particles to $\tau$ neutrinos. The excess $\tau$-lepton number is also decreased as a result [40].

Substituting Eq. (12) in (9) we obtain

$$
n_{\chi} \approx-3 K \eta_{L} \tau_{\chi} m_{\tau}^{2} \dot{T}
$$

With $\dot{T}=-T / 2 t, t \sim m_{\mathrm{P}} / T^{2}$ and $\tau_{\chi}$ from Eq. (6), this gives (omitting numerical factors)

$$
n_{\chi} \sim \frac{\eta_{L} m_{\tau}^{2}}{\alpha m_{e}^{2}} \frac{T}{m_{\mathrm{P}}} n_{\gamma}
$$

where $n_{\gamma} \sim T^{3}$ is the photon number density.

This estimate was derived assuming $T \gg m_{\tau}$, but it still applies at $T \sim m_{\tau}$. Reactions (1), (2) remain in equilibrium when $T$ drops well below $m_{\tau}$. In this regime, $\delta n_{\tau}$ and $n_{\chi}$ decrease exponentially.

Similar formulas can be written down for the decay of other unstable particles. The largest helicity is injected by the decay of the heaviest particle into the lightest particle and at the highest temperature.

\section{MAGNETIC HELICITY}

As noted in Ref. [32], the maximum magnetic helicity that can be obtained from particle helicity can be derived from the chiral anomaly equation, which can be written as a conservation law,

$$
n_{\chi}+\frac{4 \pi}{\alpha} h=\text { constant }
$$

\footnotetext{
${ }^{3}$ This estimate assumes that taus are the heaviest particles present in equilibrium at temperature $T$. If a heavier particle is present in equilibrium, it too will contribute to the mass correction and may change the estimate.
}

where $h=\langle\mathbf{A} \cdot \mathbf{B}\rangle$ is the magnetic helicity. Assuming that the initial magnetic helicity and the final particle helicity vanish, we get

$$
h_{\max }=\frac{\alpha}{4 \pi} n_{\chi} \sim \frac{\eta_{L} m_{\tau}^{2}}{4 \pi m_{e}^{2}} \frac{T}{m_{\mathrm{P}}} n_{\gamma}
$$

where we have used (14). We compare $h_{\max }$ to magnetic helicity that could be induced due to baryogenesis [3,4],

$$
h_{B} \sim \frac{\eta_{B}}{\alpha} n_{\gamma} \sim 10^{-5} \mathrm{~cm}^{-3} \sim 10^{-45} \mathrm{G}^{2} \mathrm{Mpc}
$$

where we have used the known cosmic baryon number density and are using natural units. Then

$h_{\max } \sim \frac{\eta_{L}}{\eta_{B}} \frac{\alpha m_{\tau}^{2}}{4 \pi m_{e}^{2}} \frac{T}{m_{\mathrm{P}}} h_{B} \sim 10^{-6} h_{B} \sim 10^{-51} \mathrm{G}^{2} \mathrm{Mpc}$

where we have used $T \sim 100 \mathrm{GeV}$ in the numerical estimate and the maximum allowed value $\eta_{L} \sim 10^{-2}$. Even if the decay of top quarks with mass $\sim 175 \mathrm{GeV}$ to down quarks with mass $\sim 1 \mathrm{MeV}$ is considered, $h_{\max } \sim 10^{-3} h_{B} \sim 10^{-48} \mathrm{G}^{2} \mathrm{Mpc}$.

Comparing to observations, even with the most conservative lower bound of $10^{-19} \mathrm{G}$ on Mpc scales, we get an estimate for the observed helicity $\sim 10^{-38} \mathrm{G}^{2} \mathrm{Mpc}$ which is 10 orders of magnitude larger than the most optimistic estimate above. Alternately, we can estimate the magnetic field strength on Mpc scales implied by the helicity and this gives $10^{-24} \mathrm{G}$ as compared to the observed lower bound of $10^{-19} \mathrm{G}$.

\section{CONCLUSIONS}

We have shown that the decays of certain Standard Model particles can lead to a chiral cosmological plasma around the epoch of the electroweak phase transition. The final result for the chiral asymmetry due to tau-lepton decays is given in (14). However, the asymmetry is suppressed by the lepton to photon ratio $\left(\eta_{L} \lesssim 10^{-2}\right)$ and the effect on magnetic field helicity generation is very weak as we have shown in Sec. III. Nonetheless it may be of interest that the cosmological plasma was chiral at the earliest moments even within the Standard Model of particle physics. A chiral medium may also arise in extensions of the Standard Model that include parity violating decays of very heavy particles to light Standard Model fermions at temperatures greater than $T_{\mathrm{EW}}$. Such decays would result in stronger magnetic fields with larger magnetic helicity.

\section{ACKNOWLEDGMENTS}

We thank the participants of the Nordita workshop on "Quantum Anomalies and Chiral Magnetic Phenomena," 
especially Axel Brandenburg and Kohei Kamada for feedback. We also thank Matt Baumgart, Andrew Long, Cecilia Lunardini, Igor Shovkovy, and Tracy Slatyer for discussions. The work of T. V. is supported by the U.S.
Department of Energy, Office of High Energy Physics, under Award No. DE-SC0019470 at Arizona State University. A. V. is supported by the National Science Foundation Grant No. 1820872.
[1] T. Vachaspati, Progress on cosmological magnetic fields, arXiv:2010.10525.

[2] T. Vachaspati, Magnetic fields from cosmological phase transitions. Phys. Lett. B 265, 258 (1991).

[3] J. M. Cornwall, Speculations on primordial magnetic helicity, Phys. Rev. D 56, 6146 (1997).

[4] T. Vachaspati, Estimate of the Primordial Magnetic Field Helicity, Phys. Rev. Lett. 87, 251302 (2001).

[5] M. Joyce and M.E. Shaposhnikov, Primordial Magnetic Fields, Right-Handed Electrons, and the Abelian Anomaly, Phys. Rev. Lett. 79, 1193 (1997).

[6] M. McNeil Forbes and A. R. Zhitnitsky, Primordial Galactic Magnetic Fields from Domain Walls at the QCD Phase Transition, Phys. Rev. Lett. 85, 5268 (2000).

[7] T. Stevens, M. B. Johnson, L. S. Kisslinger, and E. M. Henley, Non-Abelian Higgs model of magnetic field generation during a cosmological first-order electroweak phase transition, Phys. Rev. D 85, 063003 (2012).

[8] F. Miniati, G. Gregori, B. Reville, and S. Sarkar, AxionDriven Cosmic Magnetogenesis during the QCD Crossover, Phys. Rev. Lett. 121, 021301 (2018).

[9] R. Banerjee and K. Jedamzik, Evolution of cosmic magnetic fields: From the very early universe, to recombination, to the present, Phys. Rev. D 70, 123003 (2004).

[10] K. Jedamzik and G. Sigl, The evolution of the large-scale tail of primordial magnetic fields, Phys. Rev. D 83, 103005 (2011).

[11] A. Brandenburg, T. Kahniashvili, S. Mandal, A. R. Pol, A. G. Tevzadze, and T. Vachaspati, Evolution of hydromagnetic turbulence from the electroweak phase transition, Phys. Rev. D 96, 123528 (2017).

[12] A. Neronov and I. Vovk, Evidence for strong extragalactic magnetic fields from Fermi observations of TeV blazars, Science 328, 73 (2010).

[13] W. Essey, S. Ando, and A. Kusenko, Determination of intergalactic magnetic fields from gamma ray data, Astropart. Phys. 35, 135 (2011).

[14] K. Dolag, M. Kachelriess, S. Ostapchenko, and R. Tomas, Lower limit on the strength and filling factor of extragalactic magnetic fields, Astrophys. J. 727, L4 (2011).

[15] M. Wood, J. Biteau, R. Caputo, M. Di Mauro, and M. Meyer, Preliminary results of the Fermi high-latitude extended source catalog, Proc. Sci., ICRC2017 (2018) 647.

[16] M. Ackermann et al., The search for spatial extension in high-latitude sources detected by the Fermi large area telescope, Astrophys. J. Suppl. 237, 32 (2018).

[17] A. E. Broderick, P. Chang, and C. Pfrommer, The cosmological impact of luminous $\mathrm{TeV}$ blazars I: Implications of plasma instabilities for the intergalactic magnetic field and extragalactic gamma-ray background, Astrophys. J. 752, 22 (2012).

[18] T. C. Arlen, V. V. Vassiliev, T. Weisgarber, S. P. Wakely, and S. Yusef Shafi, Intergalactic magnetic fields and gamma ray observations of extreme TeV blazars, Astrophys. J. 796, 18 (2014).

[19] W. Chen, J. H. Buckley, and F. Ferrer, Search for GeV? Ray Pair Halos around Low Redshift Blazars, Phys. Rev. Lett. 115, 211103 (2015).

[20] H. Tashiro, W. Chen, F. Ferrer, and T. Vachaspati, Search for $C P$ violating signature of intergalactic magnetic helicity in the gamma ray sky, Mon. Not. R. Astron. Soc. 445, L41 (2014).

[21] W. Chen, B. D. Chowdhury, F. Ferrer, H. Tashiro, and T. Vachaspati, Intergalactic magnetic field spectra from diffuse gamma rays, Mon. Not. R. Astron. Soc. 450, 3371 (2015).

[22] J. Asplund, G. Johannesson, and A. Brandenburg, On the measurement of handedness in Fermi Large Area Telescope data, Astrophys. J. 898, 124 (2020).

[23] M. Kachelriess and B. C. Martinez, Searching for primordial helical magnetic fields, Phys. Rev. D 102, 083001 (2020).

[24] D. E. Kharzeev, The chiral magnetohydrodynamics of QCD fluid at RHIC and LHC, J. Phys. G 38, 124061 (2011).

[25] A. Vilenkin, Equilibrium parity violating current in a magnetic field, Phys. Rev. D 22, 3080 (1980).

[26] A. Vilenkin, Macroscopic parity violating effects: Neutrino fluxes from rotating black holes and in rotating thermal radiation, Phys. Rev. D 20, 1807 (1979).

[27] A. Boyarsky, J. Frohlich, and O. Ruchayskiy, Self-Consistent Evolution of Magnetic Fields and Chiral Asymmetry in the Early Universe, Phys. Rev. Lett. 108, 031301 (2012).

[28] H. Tashiro, T. Vachaspati, and A. Vilenkin, Chiral effects and cosmic magnetic fields, Phys. Rev. D 86, 105033 (2012).

[29] M. Dvornikov and V. B. Semikoz, Lepton asymmetry growth in the symmetric phase of an electroweak plasma with hypermagnetic fields versus its washing out by sphalerons, Phys. Rev. D 87, 025023 (2013).

[30] M. Dvornikov and V. B. Semikoz, Instability of magnetic fields in electroweak plasma driven by neutrino asymmetries, J. Cosmol. Astropart. Phys. 05 (2014) 002.

[31] M. Dvornikov and V. B. Semikoz, Influence of the turbulent motion on the chiral magnetic effect in the early universe, Phys. Rev. D 95, 043538 (2017).

[32] A. Brandenburg, J. Schober, I. Rogachevskii, T. Kahniashvili, A. Boyarsky, J. Frohlich, O. Ruchayskiy, and N. Kleeorin, The turbulent chiral-magnetic cascade in the early universe, Astrophys. J. 845, L21 (2017). 
[33] Y. Masada, K. Kotake, T. Takiwaki, and N. Yamamoto, Chiral magnetohydrodynamic turbulence in core-collapse supernovae, Phys. Rev. D 98, 083018 (2018).

[34] D. Grabowska, D. B. Kaplan, and S. Reddy, Role of the electron mass in damping chiral plasma instability in supernovae and neutron stars, Phys. Rev. D 91, 085035 (2015).

[35] D. G. Figueroa and M. Shaposhnikov, Anomalous nonconservation of fermion/chiral number in Abelian gauge theories at finite temperature, J. High Energy Phys. 04 (2018) 026.

[36] A. Boyarsky, V. Cheianov, O. Ruchayskiy, and O. Sobol, Evolution of the Primordial Axial Charge across Cosmic Times, Phys. Rev. Lett. 126, 021801 (2021).
[37] V. A. Kuzmin, V. A. Rubakov, and M. E. Shaposhnikov, Anomalous electroweak baryon number nonconservation and GUT mechanism for baryogenesis, Phys. Lett. B 191, 171 (1987).

[38] J. A. Harvey and M. S. Turner, Cosmological baryon and lepton number in the presence of electroweak fermion number violation, Phys. Rev. D 42, 3344 (1990).

[39] D. J. Schwarz and M. Stuke, Does the CMB prefer a leptonic Universe? New J. Phys. 15, 033021 (2013).

[40] A. I. Bochkarev, S. Yu. Khlebnikov, and M. E. Shaposhnikov, Sphalerons and baryogenesis: Electroweak $C P$ violation at high temperatures, Nucl. Phys. B329, 493 (1990). 